\title{
Familiarity influences odor memory stability
}

\author{
Richard J. Stevenson • Mehmet K. Mahmut
}

Published online: 24 January 2013

(C) Psychonomic Society, Inc. 2013

\begin{abstract}
It has been suggested that olfactory perception relies on a memory-based pattern-matching system. In this experiment, we tested a prediction derived from this approach, namely that representations of unfamiliar odors are less stable than those of familiar odors. Participants provided qualitative descriptions of odors either immediately after smelling them or after a 1- or 3-min delay. Later, participants were given a surprise test in which they were asked to match their earlier descriptions to those same odors. Delay exerted no effect on familiar odors. However, for unfamiliar odors, while their matching performance was equivalent to that for familiar odors when descriptions were made immediately, delay significantly impaired performance. The better capacity to name familiar odors only assisted matching performance at the longest (3-min) delay. These findings suggest that unfamiliar odors have less stable representations than do familiar odors. Pattern-matching theory suggests that this occurs because unfamiliar odors weakly activate many nodes in memory, resulting in less-stable percepts.
\end{abstract}

Keywords Odor · Pattern matching · Olfactory cognition . Familiarity

\section{Introduction}

Perceptual judgments in olfaction are heavily influenced by experience. The abilities to discriminate one odor from another and to detect an odor in a mixture are improved if the target is familiar (e.g., Rabin, 1988), while repeatedly experiencing two odorants together acts to make them less discriminable (e.g., Stevenson, 2001). In participants who have little experience with a particular class of odors (e.g., wines) or with odors in

R. J. Stevenson $(\bowtie) \cdot$ M. K. Mahmut

Department of Psychology, Macquarie University, Sydney,

New South Wales 2109, Australia

e-mail: dick.stevenson@mq.edu.au general (e.g., children), relevant odor discrimination tasks are performed more poorly than among those with greater experience (e.g., Melcher \& Schooler, 1996; Stevenson, Mahmut, \& Sundqvist, 2007). A mnemonic basis for these effects is suggested by observations from neuropsychology, as damage to the integrity of olfactory memory impairs the capacity to tell one odor from another (e.g., Eichenbaum, Morton, Potter, \& Corkin, 1983). Together, these and other findings have led to the suggestion that olfactory perception relies on pattern matching, with the input from receptors being matched in a contentaddressable memory store that contains previously encountered odor patterns (for variants of this basic idea, see Bower, 1991; Haberly, 2001; Lynch, 1986; Malnic, Hirono, Sato, \& Buck, 1999; Stevenson \& Wilson, 2007). In this study, we tested a novel prediction derived from this pattern-matching approach to olfaction.

Unfamiliar odors have been found to be more redolent of other smells than are familiar odors, and it has been suggested that this results from the pattern-matching process described above (Mingo \& Stevenson, 2007). A familiar odor may activate only a limited number of highly similar matches in content-addressable memory (CAM), an effect that may be strengthened by the ability of strong matches to inhibit weaker matches (see Stevenson \& Boakes, 2003). Unfamiliar odors, which have no true match, may partially activate many memories in CAM, without any inhibition occurring from stronger matches. One consequence of this is that unfamiliar odors smell somewhat similar to many other odors, with this being a presumed phenomenological correlate of their poorer discriminability relative to familiar odors (e.g., Rabin, 1988). Another consequence of partially activating many matches is that the perceptual representation of an unfamiliar odor should be less stable than that of a familiar odor. Assuming a constant decay rate, unfamiliar odors should be more vulnerable to activation decay than are familiar odors, as unfamiliar odors start the decay process from a far lower activation baseline. So while an unfamiliar odor may remind one of many odors, this percept 
may be less stable than that of a familiar odor once the stimulus is withdrawn. In the experiment reported here, we set out to test this novel prediction.

To determine whether the loss of information over time is greater for unfamiliar than for familiar odors, the following procedure was adopted. Participants were asked to smell a set of odors. For each odor, they were asked to describe what it smelled like, using multiple redolence ratings (e.g., "how lemon-like does this smell?"). Later, without knowing that this would happen, participants were handed all of their earlier redolence ratings and the associated odors, and they were asked to match one to the other. The key manipulation here was to vary the interval between smelling the odor and rating it on the multiple redolence ratings. For one set of odors, participants smelled each odor and then immediately completed their redolence ratings. For a second set, they smelled the odor and then waited $1 \mathrm{~min}$ before completing their redolence ratings. For a third set, they smelled each odor and then waited 3 min before completing the redolence ratings. We reasoned that because unfamiliar odors start activation decay from a low baseline, decay across a delay should adversely affect the quality of the participants' odor descriptions (i.e., redolence ratings), and poorer-quality descriptions should then be harder to match to their corresponding odors. In contrast, if activation decay starts from a much higher baseline for familiar odors, activation decay across a delay should have less impact on the quality of the description provided, and hence on later matching performance.

We also asked participants, in addition to completing redolence ratings, to make other judgments about each odor. They judged familiarity, so that we had a basis for deciding whether a particular odor was familiar or unfamiliar to that person. We also asked them to judge how complex that they thought the odor smelled, how much they liked (or disliked) it, how intensely it smelled, and whether they knew its name. This last question was particularly important. Although odor naming is generally poor, familiar odors are correctly named more frequently than unfamiliar odors (e.g., Cain, 1979). If smelling a familiar odor leads to the generation of a name, the name might then compensate for any activation decay that might occur. This could lead, erroneously, to the conclusion that familiar odors do indeed differ in activation decay from unfamiliar odors, when in fact the difference between familiar and unfamiliar odors results solely from superior naming. Determining which odors were named and which were not enabled us to test whether this variable affected any observed difference in matching performance between the familiar and unfamiliar odors.

Finally, on the basis of the redolence ratings, we also calculated the number of odors that participants claimed that each target reminded them of and the degree to which the components reminded them of the odor. From past research (e.g., Distel et al., 1999; Mingo \& Stevenson, 2007), we expected unfamiliar odors, relative to familiar odors, to be judged as being less intense, less pleasant, and redolent of more odors but to a lesser extent, and also to be named less often. Although this had not been assessed before, we also expected unfamiliar odors to be judged as being more complex than familiar smells. To recap, this experiment was planned to test whether representations of unfamiliar odors are less stable than those of familiar odors, as predicted by memory-based pattern-matching models of olfactory perception.

\section{Method}

\section{Participants}

A group of 47 healthy participants (mean age $=20.9, S D=$ $5.8 ; 35$ female, 12 male) successfully completed the experiment. Written informed consent was obtained from each participant, and the protocol was approved by the Macquarie University Human Research Ethics committee.

\section{Stimuli}

Odorants were applied (in quantities and from sources indicated in Table 1) to cotton balls and then placed in opaque 250-ml plastic squeezy bottles. Three sets of odorants, each composed of ten stimuli, were used, and these are detailed in Table 1.

\section{Procedure}

The experiment was composed of two parts. In the first part, participants made ratings of the three sets of odors (30 in total), with each odor constituting a trial. One of the sets of ten odors was always rated immediately after smelling was complete, another of the sets of ten odors was always rated following a 1-min delay after smelling was complete, and the third set of ten odors was always rated following a 3-min delay after smelling was complete. For each participant, one odor set was allocated to each delay type, and this allocation was counterbalanced across participants. The presentation order of the 30 trials was randomized separately for each participant, so that any delay type (i.e., immediate, $1 \mathrm{~min}$, and $3 \mathrm{~min}$ ) could be followed by any other delay type.

On each trial, the experimenter administered the odorant by holding the snout of the squeezy bottle $7 \mathrm{~cm}$ underneath the participant's nostrils. Participants were then asked to sniff while the experimenter squeezed the bottle three times. Immediately after sniffing had finished, after a 1-min delay (sitting quietly), or after a 3-min delay (sitting quietly) participants were asked to complete two sheets of ratings. 
Table 1 The three sets of odorants used in the experiment

\begin{tabular}{|c|c|c|}
\hline Floral Set & Fruit Set & Chemical Set \\
\hline $\begin{array}{l}\text { Name } \\
\text { (quantity, source) }\end{array}$ & $\begin{array}{l}\text { Name } \\
\text { (quantity, source) }\end{array}$ & $\begin{array}{l}\text { Name } \\
\text { (quantity, source) }\end{array}$ \\
\hline $\begin{array}{l}\text { Vetiver } \\
\text { (0.05 g; Ess. oil) }\end{array}$ & $\begin{array}{l}\text { Cupuacu } \\
\quad(0.1 \mathrm{~g} ; \text { Dragoco })\end{array}$ & $\begin{array}{l}\text { Turps } \\
\text { (1.0 g; Homebrand) }\end{array}$ \\
\hline $\begin{array}{l}\text { Angelica } \\
\text { (0.05 g; Ess. oil) }\end{array}$ & $\begin{array}{l}\text { Guava } \\
\text { (0.07 g; Dragoco) }\end{array}$ & $\begin{array}{l}\text { Lighter fluid } \\
\quad(0.15 \mathrm{~g} ; \text { Zippo })\end{array}$ \\
\hline $\begin{array}{l}\text { Ylang ylang } \\
\text { (0.05 g; Ess. oil) }\end{array}$ & $\begin{array}{l}\text { Longan } \\
\qquad(0.13 \mathrm{~g} ; \text { Dragoco })\end{array}$ & $\begin{array}{l}\text { Boot polish } \\
(2.5 \mathrm{~g} \text {; Kiwi })\end{array}$ \\
\hline $\begin{array}{l}\text { Patchouli } \\
\quad(0.05 \text { g; Ess. oil })\end{array}$ & $\begin{array}{l}\text { W. chestnut } \\
\quad(0.07 \mathrm{~g} \text {; Quest })\end{array}$ & $\begin{array}{l}\text { Butanol } \\
\quad(0.05 \mathrm{~g} ; \text { Sigma })\end{array}$ \\
\hline $\begin{array}{l}\text { Lavender } \\
\qquad(0.05 \text { g; Ess. oil })\end{array}$ & $\begin{array}{l}\text { Lychee } \\
\text { (0.07 g; Quest) }\end{array}$ & $\begin{array}{l}\text { Acetephenone } \\
\text { (0.03 g; Sigma) }\end{array}$ \\
\hline $\begin{array}{l}\text { Rose water } \\
\quad(0.5 \mathrm{~g} \text {; Essence })\end{array}$ & $\begin{array}{l}\text { Cherry } \\
(0.07 \mathrm{~g} ; \text { Quest })\end{array}$ & $\begin{array}{l}\text { Naphthalene } \\
\text { (0.3 g; Sigma) }\end{array}$ \\
\hline $\begin{array}{l}\text { Jasmine } \\
\quad(0.05 \mathrm{~g} ; \text { Ess. oil })\end{array}$ & $\begin{array}{l}\text { Grape } \\
\quad(0.15 \mathrm{~g} ; \text { Quest })\end{array}$ & $\begin{array}{l}\text { Octanol } \\
\text { (0.1 g; Sigma) }\end{array}$ \\
\hline $\begin{array}{l}\text { Ionone } \\
\quad(0.07 \mathrm{~g} \text {; Sigma })\end{array}$ & $\begin{array}{l}\text { Kiwifruit } \\
\quad \text { (0.1 g; Dragoco) }\end{array}$ & $\begin{array}{l}\text { Terpineol } \\
\text { (0.1 g; Sigma) }\end{array}$ \\
\hline $\begin{array}{l}\text { Anise } \\
\text { (0.15 g; Dragoco })\end{array}$ & $\begin{array}{l}\text { Pear } \\
\quad(0.15 \mathrm{~g} ; \text { Quest })\end{array}$ & $\begin{array}{l}p \text {-Anisaldehyde } \\
(0.1 \mathrm{~g} ; \text { Sigma })\end{array}$ \\
\hline $\begin{array}{l}\text { Citral } \\
\quad(0.025 \mathrm{~g} ; \text { Dragoco })\end{array}$ & $\begin{array}{l}\text { Banana } \\
\text { (0.1 g; Quest) }\end{array}$ & $\begin{array}{l}\text { Guaicol } \\
\text { (0.05 g; Sigma). }\end{array}$ \\
\hline
\end{tabular}

Ess. oil, essential oil; W. chestnut, Water chestnut

The first sheet - the redolence ratings - was always particular to the odorant set from which the trial odor had been drawn and was composed of 19 descriptors pertinent to that particular set (see Appendix A). Participants were asked to rate how applicable each of the 19 descriptors was for that odor, using seven-point category scales (anchors: absent [0], to slightly [1], to moderately [3], to extremely [6]; after Dravnieks, 1985). Once all of the ratings were complete, the sheet was removed from view and participants were presented with a second rating sheet that was identical for all odors. Here, participants evaluated, using other sevenpoint category scales, how much they liked the odor (anchors: dislike [0], indifferent [3], like [6]), how complex they thought that it smelled (anchors: simple [0], moderate [3], very [6]), how familiar they were with the smell (anchors: never smelled before today [0], smelled a few times before [3], smelled many times before [6]), and how strongly it smelled (anchors: absent [0], slightly [1], moderately [3], extremely [6]). Finally, participants were asked to name the odor, or to guess if no name came to mind (R.J.S. later performed the name coding, basing each decision on its closeness to the veridical name). After all of the ratings had been completed, the next trial commenced.

The second part of the experiment was composed of three surprise matching tasks, one for each odor set (i.e., delay interval), presented in counterbalanced order (i.e., each odor set was as likely to be matched first as any other). Each matching test was conducted in the same manner: All of the odors from one set (e.g., the floral set) were placed in front of the participant. The participant was then handed the ten redolence-rating sheets in the same random order that they had been completed for these ten odors during Part 1 of the experiment. The participant was then asked to match, as best he or she could, each redolence-rating sheet to each odor. This test was self-paced, and they were allowed to smell, shift, and change their matches as many times as they liked, until they were satisfied with their selections. This process was then repeated for the other odor sets.

\section{Analysis}

Each participant's data were categorized into familiar and unfamiliar odor groupings in the following way. For a given test delay (i.e., immediate, $1 \mathrm{~min}$, or $3 \mathrm{~min}$ ), the relevant set of odors were first ranked by their accompanying familiarity rating. This could result in the ten odors being potentially assigned to between one (i.e., all ten odors given the same familiarity score) and seven groupings (i.e., within the ten odors, seven were given different familiarity ratings). Across all 47 participants and the three delay intervals (i.e., three odor sets) - that is, on the 141 occasions in which an odor set had to be ranked by familiarity - the actual grouping sizes varied from two to seven, which occurred with the following frequencies: two familiarity groupings, $0.7 \%$; three, $12.0 \%$; four, $29.8 \%$; five, $36.9 \%$; six, $16.3 \%$; and seven, $4.3 \%$. We then used the following rules to assign odors within a particular set to a "familiar" or "unfamiliar" group: (1) When there were just two groupings, both were retained; (2) with three groupings, the middle group was dispensed with; (3) with four groupings, the two lowest were combined, as were the two highest; (4) with five groupings, the middle grouping was dispensed with, and the highest two groups and lowest two groups were each combined; (5) with six groupings, the middle two groupings were dispensed with and the highest and lowest each combined; and (6) with seven groupings, the middle three groupings were dispensed with and the highest and lowest each combined. These categorization rules ensured that the familiar and unfamiliar odor groupings maximally differed in familiarity, while also ensuring that there would be sufficient and similar numbers of familiar and unfamiliar odor trials available for analysis. With regard to this last point, the numbers of familiar $(M=12.3 / 30)$ and unfamiliar $(M=$ $12.9 / 30$ ) odor trials retained for analysis did not differ, nor did the groups differ across delay intervals (all $F_{\mathrm{S}}<1$; two-way repeated measures analysis of variance [ANOVA] for familiarity by delay interval). The percentages of correct matches for the unfamiliar and familiar odor groups at each delay interval were then calculated for each participant, as were 
the means for the various judgments made during the rating phase (familiarity, intensity, complexity, etc.).

\section{Results}

\section{Matching performance}

The mean matching performance for the low- and highfamiliarity odor groupings that were rated immediately or following a 1-min and a 3-min delay are illustrated in Fig. 1. As some of these data were skewed, some were kurtotic, and some both, they were analyzed nonparametrically. We tested for main effects of familiarity (familiar vs. unfamiliar) by collapsing across delays, and of delay ( 0 vs. 1 vs. $3 \mathrm{~min}$ ) by collapsing across familiarity conditions. For the interaction between familiarity and delay, we compared the difference between familiar and unfamiliar matching performance by delays. Two significant effects emerged from this analysis. First, a significant effect of familiarity (Wilcoxon test, $Z=4.15, p<.001)$, with better matching of familiar $(M=40.1 \%)$ than of unfamiliar $(M=23.1 \%)$ odors, and second, a significant interaction between delay and familiarity [Friedman test, $\chi^{2}(2)=7.54, p<.025$ ]; see Fig. 1. Bonferronicorrected comparisons (Wilcoxon tests) revealed that the familiar and unfamiliar odor groupings did not differ in the immediate condition but did in the 1-min $(Z=3.85, p<.017)$ and 3-min $(Z=3.34, p<.017)$ delay conditions. We also checked to see whether matching performance would decline from the immediate to the delayed testing conditions in the unfamiliar and familiar groupings, using Page's test for ordered alternatives.

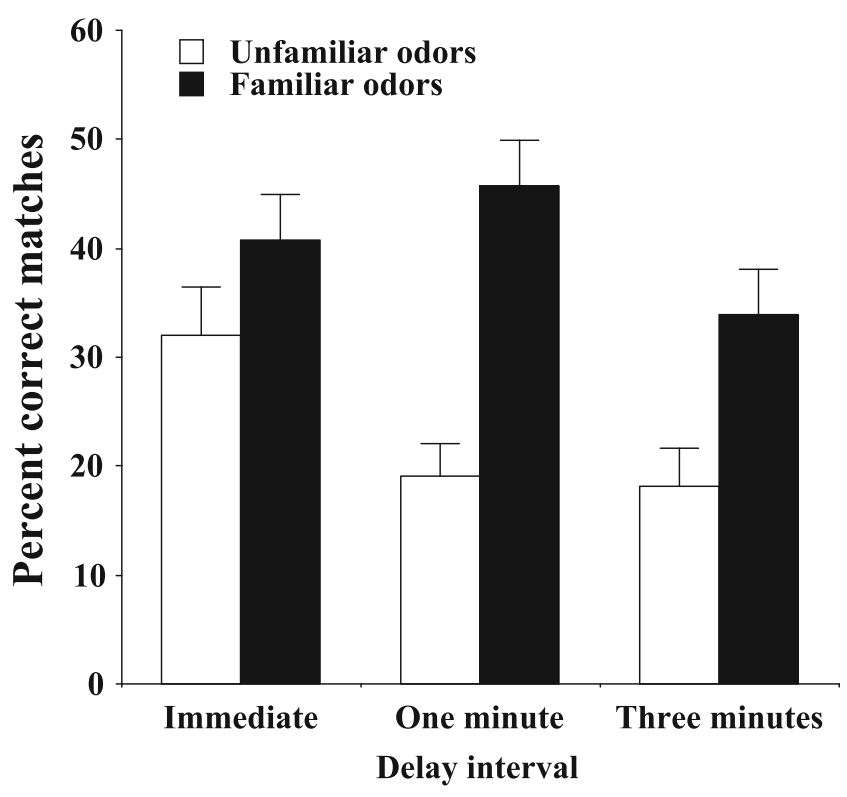

Fig. 1 Mean percentages of correct matches (and standard errors) for familiar and unfamiliar odors, when ratings used for the matching task were completed either immediately or after a 1-min or 3-min delay
Matching scores decreased significantly across delays for the unfamiliar odor groupings $(Z=2.58, p<.005)$, but not for the familiar odor groupings. These findings indicate that delay had a significant impact on the quality of the reports that participants made for unfamiliar odors, but not for familiar odors. Moreover, delay was associated with a loss of descriptive quality for unfamiliar, but not familiar, odors.

Characteristics of the familiar and unfamiliar odors

Familiarity, intensity, and liking ratings, as well as the number of selected odors that the target reminded the judge of (redolence), the mean applicability of each of these reminders (applicability), and the apparent complexity of the odor, were all analyzed using two-way ANOVAs with Familiarity (familiar vs. unfamiliar) and Delay (0 vs. 1 vs. $3 \mathrm{~min}$ ) as withinparticipants factors. In each of these analyses, only the main effect of familiarity was significant. The statistics associated with each analysis and the means for the two familiarity groupings are all presented in Table 2. Unfamiliar odors were - as expected - judged to be more unfamiliar than the familiar odor groupings. In addition, the unfamiliar odors were liked less, judged as being less intense, and rated as being more complex and more redolent of other smells, but with lower mean applicability ratings for each of the reminders. The naming data were skewed and kurtotic, and so were analyzed nonparametrically, testing for all of the effects examined in the ANOVA design above. The only effect to emerge was that of familiarity (Wilcoxon test, $Z=5.68, p<.001$ ), with better naming for the familiar ( $M=30.0 \%$ named) than for the unfamiliar $(M=$ $5.0 \%$ named) odor groupings.

\section{Effect of naming}

As odor naming was consistently better overall for familiar odors, we wished to determine its effect on matching performance. To do this, we grouped participants at each level of delay into one of two naming groups: (1) The equal-naming group consisted of participants who were as good at naming the unfamiliar odors as at naming the familiar odors, and (2) the familiar-naming group consisted of those who were better at naming the familiar than the unfamiliar odors. The next step involved calculating the matching performance difference scores between familiar and unfamiliar matching performance at each level of delay (i.e., the difference between the bars at each delay in Fig. 1). We then tested, again at each level of delay, whether the sizes of the matching performance difference scores significantly differed for the naming groups. No significant differences emerged at the 0 - or the 1-min delay (i.e., the data in both naming groups looked identical to those in Fig. 1). However, at the 3-min delay, for the equal-naming group (i.e., when familiar and unfamiliar odors were named equally well), we found no difference in matching performance (mean 
Table 2 Mean characteristics (and standard deviations) of the familiar and unfamiliar odors

\begin{tabular}{|c|c|c|c|c|c|}
\hline \multirow[b]{2}{*}{ Variable } & \multicolumn{2}{|c|}{ Odor Groupings } & \multicolumn{3}{|c|}{ Familiarity Main Effect } \\
\hline & Familiar & Unfamiliar & $F(1,46)=$ & $M S E=$ & Partial eta ${ }^{2}$ \\
\hline Familiarity & $4.6(0.7)$ & $1.4(0.6)$ & $917.14^{*}$ & 0.80 & .95 \\
\hline Complexity & $2.8(0.8)$ & $3.2(0.8)$ & $5.38^{*}$ & 1.44 & .11 \\
\hline Redolence & $5.4(3.3)$ & $5.9(3.1)$ & $6.62^{*}$ & 1.99 & .13 \\
\hline Applicability & $3.3(0.8)$ & $2.5(0.7)$ & $73.60^{*}$ & 0.68 & .62 \\
\hline Liking & $3.4(0.8)$ & $2.3(0.9)$ & $60.98^{*}$ & 1.26 & .57 \\
\hline Intensity & $3.9(0.6)$ & $3.2(0.7)$ & $48.62^{*}$ & 0.77 & .51 \\
\hline
\end{tabular}

${ }^{*} p<.05$

difference $=-1.0$ ), but in the familiar naming group, matching was better for familiar odors (mean difference $=26.2 ; Z=3.04$, $p<.002$ ). Thus, at the 3-min delay, naming familiar odors was associated with better matching performance.

\section{Discussion}

The aim of this experiment was to investigate a novel prediction derived from a memory-based model of olfactory perception: namely, whether unfamiliar odor representations are less stable than those for familiar odors. As expected, we found that the quality of descriptions for familiar odors did not change when an interval was introduced between smelling the odor and making the redolence ratings. However, for unfamiliar odors, although the descriptions obtained immediately after smelling were as good as those obtained for familiar odors, delay adversely affected the quality of these reports. Descriptions obtained at 1- and 3-min intervals for unfamiliar odors were less able to support matching, implying that they were of poorer quality than (1) those obtained from familiar odors at comparable delays and (2) those obtained from unfamiliar odors immediately after smelling the odor. While this difference in matching performance for familiar and unfamiliar odors across delays could not be accounted for by differences in odor naming at the 1-min delay, it could at the 3-min delay. Here, participants who could name the familiar odors were able to generate descriptions that were of higher quality than those from unfamiliar odors. We also expected to find several phenomenological differences between familiar and unfamiliar odors. In line with our prior findings, we observed that unfamiliar odors were judged to be redolent of more odors, although they matched each one to a lesser extent than did familiar odors (Mingo \& Stevenson, 2007). In addition, unfamiliar odors were judged as smelling more complex, less intense, and less pleasant than familiar odors - the latter two findings also replicating prior work (e.g., Distel et al., 1999). Finally, as had been observed in other matching studies, performance was generally quite poor on this task, presumably because of the difficulty that participants have in accurately using words to describe their olfactory experience (e.g., Lawless, 1984).

The matching data here support the idea that the representation of an unfamiliar odor is less stable than that of a familiar odor. This conclusion seems to concur with the findings from a study by Rabin and Cain (1984), in which odor recognition memory performance was significantly poorer for unfamiliar odors. These effects are presumed to occur because many nodes in odor memory somewhat resemble the pattern generated by an unfamiliar odor. As these matches are only partial, the resulting activations are weak, so that the effects of activation decay are observed more rapidly than for familiar odors, even though decay is hypothesized to occur at the same rate. This results in poorer-quality descriptions of olfactory experience following a delay, and thus in poorer matching performance. In contrast, familiar odors are argued to match fewer memory nodes, with a stronger match to each node, particularly for those that an odor most closely resembles (i.e., previous encounters with that odor). This results in greater initial activation, providing for a more stable representation across time, making delay a less pressing issue than it is for unfamiliar odors. These findings are consistent with the type of memorybased pattern-matching models of olfactory perception that we outlined in the introduction (i.e., Bower, 1991; Haberly, 2001; Lynch, 1986; Malnic et al., 1999; Stevenson \& Wilson, 2007).

Our data also indicated that after a 3-min delay, participants found it hard to generate an accurate description of a familiar odor, unless they were able to correctly name the smell when they sampled it. Two conclusions arise from this observation. This finding suggests, first, that activation decay to a level that will not support the generation of an accurate description must be complete for all odors by 3 min postsmelling, and second, that naming provides - as has previously been demonstrated in a number of studies - an additional means (other than memory activation, as described here) of preserving olfactory information across temporal delays (e.g., Herz, 2000; Jönsson, Møller, \& Olsson, 2011). Considerable debate has focused on the nature of olfactory short-term memory, especially concerning the role of verbal processes. We have argued elsewhere that 
short- and long-term retention of odors may both rely on a common odor perceptual memory system (e.g., Stevenson \& Boakes, 2003). Short-term retention is presumed to be supported by the activation of long-term memory nodes, with activation below the level required for a conscious representation providing the basis for many laboratory olfactory shortterm memory tasks (especially recognition). This view predicts no difference in perceptual codes between the short- and longterm stores, as well as suggesting that specific deficits in olfactory short-term memory will not be observed in neuropsychological populations. Both of these points are consistent with current knowledge (White, 2009). Finally, this perceptual memory system can be complemented by verbal memory processes - as we found here, and as was seen by other investigators studying olfactory memory (e.g., Lyman \& McDaniel, 1990).

In conclusion, few studies have tested the predictions derived from memory-based approaches to human olfactory perception. In the experiment reported here, we tested one prediction derived from this approach, finding that unfamiliar odor representations are indeed less stable than those of familiar odors. This may result from the fact that unfamiliar odors activate more nodes in odor memory, but all to a lesser degree than do familiar odors, leading the former activation to decay more rapidly across time. This new finding is consistent with a memory-based pattern-matching account of human olfactory perception.

\section{Appendix A}

Table 3 Redolence ratings used for each odor set

\begin{tabular}{lll}
\hline Floral Set & Chemical Set & Fruit Set \\
\hline Minty & Turps & Melon \\
Orange blossom & Disinfectant & Prunes \\
Oregano & Plastic & Strawberry \\
Pungent & Fishy & Banana \\
Liquorice & Papery & Pineapple \\
Female perfume & Wet clothes & Peach \\
Lavender & Fatty & Blueberry \\
Musk & Varnish & Raisins \\
Lilac & Solvent & Grape \\
Jasmine & Soapy & Orange \\
Cinnamon & Medicinal & Lychee \\
Woody & Sulphurous & Cherry \\
Male perfume & Mothballs & Apple \\
Lemon blossom & Tar & Lemon \\
Faecal & Petrol & Dried fruit \\
Violets & Rubbery & Jam \\
Cloves & Paint & Pear \\
Cut grass & Burnt match & Grapefruit \\
Rose & Marker pen & Kiwi \\
\hline
\end{tabular}

\section{References}

Bower, J. M. (1991). Piriform cortex and olfactory object recognition. In J. Davis \& H. Eichenbaum (Eds.), Olfaction as a model system for computational neuroscience (pp. 265-285). Cambridge, MA: MIT Press.

Cain, W. (1979). To know with the nose: Keys to odor identification. Science, 203, 467-470.

Distel, H., Ayabe-Kanamura, S., Schicker, I., Martinez-Gomez, M., Kobayakawa, T., Saito, S., \& Hudson, R. (1999). Perceptions of everyday odors - Correlations between intensity, familiarity and strength of hedonic judgment. Chemical Senses, 24, 191199.

Dravnieks, A. (1985). Atlas of odor character profiles. Philadelphia, PA: American Society for Testing and Materials.

Eichenbaum, H., Morton, T. H., Potter, H., \& Corkin, S. (1983). Selective olfactory deficits in case H.M. Brain, 106, 459472.

Haberly, L. B. (2001). Parallel-distributed processing in olfactory cortex: New insights from morphological and physiological analysis of neuronal circuitry. Chemical Senses, 26, 551577.

Herz, R. (2000). Verbal coding in olfactory versus nonolfactory cognition. Memory and Cognition, 28, 957-964.

Jönsson, F. U., Møller, P., \& Olsson, M. J. (2011). Olfactory working memory: Effects of verbalization on the 2-back task. Memory and Cognition, 39, 1023-1032. doi:10.3758/s13421011-0080-5

Lawless, H. (1984). Flavour description of white wine by "expert" and non expert wine consumers. Journal of Food Science, 49, 120 123.

Lyman, B., \& McDaniel, M. (1990). Memory for odors and odor names: Modalities of elaboration and imagery. Journal of Experimental Psychology: Learning, Memory, and Cognition, $38,753-765$.

Lynch, G. (1986). Synapses, circuits and the beginnings of memory. Cambridge, MA: MIT Press.

Malnic, B., Hirono, J., Sato, T., \& Buck, L. B. (1999). Combinatorial receptor codes for odors. Cell, 96, 713-723.

Melcher, J. M., \& Schooler, J. W. (1996). The misremembrance of wines past: Verbal and perceptual expertise differentially mediate verbal overshadowing of taste memory. Journal of Memory and Language, 35, 231-245. doi:10.1006/jmla.1996.0013

Mingo, S., \& Stevenson, R. J. (2007). Phenomenological differences between familiar and unfamiliar odours. Perception, 36, 931-947.

Rabin, M. D. (1988). Experience facilitates olfactory quality discrimination. Perception \& Psychophysics, 44, 532-540.

Rabin, M. D., \& Cain, W. S. (1984). Odor recognition: Familiarity, identifiability, and encoding consistency. Journal of Experimental Psychology: Learning, Memory, and Cognition, 10, 316-325.

Stevenson, R. J. (2001). Perceptual learning with odors: Implications for psychological accounts of odor quality perception. Psychonomic Bulletin and Review, 8, 708-712.

Stevenson, R. J., \& Boakes, R. A. (2003). A mnemonic theory of odor perception. Psychological Review, 110, 340-364.

Stevenson, R. J., Mahmut, M., \& Sundqvist, N. (2007). Age-related changes in odor discrimination. Developmental Psychology, 43, 253-260.

Stevenson, R. J., \& Wilson, D. A. (2007). Olfactory perception: An object recognition approach. Perception, 36, 1821-1833.

White, T. L. (2009). A second look at the structure of human olfactory memory. Annals of the New York Academy of Sciences, 1170, $338-342$. 